\title{
Association between Sleep Disturbances and Abdominal Symptoms
}

\author{
Yoshiya Morito ${ }^{1}$, Masahito Aimi ${ }^{1}$, Norihisa Ishimura ${ }^{1}$, Shino Shimura ${ }^{1}$, Hironobu Mikami ${ }^{1}$, \\ Eiko Okimoto ${ }^{1}$, Shuuichi Sato ${ }^{1}$, Shunji Ishihara ${ }^{1}$, Yoshinori Kushiyama ${ }^{2}$, Tomoko Katsube ${ }^{3}$, \\ Kyoichi Adachi ${ }^{4}$ and Yoshikazu Kinoshita ${ }^{1}$
}

\begin{abstract}
Objective Although gastroesophageal reflux disease (GERD) is known to cause sleep disturbances, the relationships between other abdominal symptoms and sleep disorders have not been clarified. In the present study, we examined the relationships between daytime sleepiness and various abdominal symptoms in a nonclinical population.

Methods We enrolled 2,936 subjects who visited Matsue Red Cross Hospital for an annual health check examination during a 10-month consecutive period after excluding those with organic gastrointestinal diseases. The Izumo scale abdominal symptom and Epworth Sleepiness Scale (ESS) questionnaires were employed to evaluate the presence of abdominal symptoms and daytime sleepiness.

Results Among the 2,936 subjects, 233 (7.9\%), 254 (8.6\%) and 528 (18\%) had GERD-like, functional dyspepsia (FD)-like and irritable bowel syndrome (IBS)-like symptoms, respectively. The ESS scores in the subjects with GERD-, FD- and IBS-like symptoms were significantly higher than those observed in the asymptomatic subjects. The subjects with multiple abdominal symptoms tended to have higher ESS scores than those with single symptoms. A multiple logistic regression analysis revealed a younger age and the presence of FD- and IBS-like symptoms to be significant influencing factors for sleep disturbances.

Conclusion The presence of FD and IBS symptoms in addition to GERD symptoms exhibits a strong relationship with sleep disturbances from the viewpoint of daytime sleepiness.
\end{abstract}

Key words: abdominal symptoms, sleep disturbance, Izumo scale, Epworth Sleeping Scale

(Intern Med 53: 2179-2183, 2014)

(DOI: 10.2169/internalmedicine.53.2591)

\section{Introduction}

Good sleep is considered to be important for the quality of life, and many pathological conditions, including mental stress, are known to decrease the quality of sleep. Patients with gastroesophageal reflux disease (GERD) are reported to have a decreased quality of sleep due to heartburn and/or acid regurgitation disturbances $(1,2)$. Indeed, treatment of GERD with the administration of a proton pump inhibitor (PPI) has been shown to improve not only reflux symptoms, but also sleep quality (3-6). However, the effects of abdominal symptoms other than heartburn and acid regurgitation on sleep have not been thoroughly investigated. Recently, dyspepsia was reported to decrease sleep quality, similar to reflux symptoms; however, information regarding other abdominal symptoms is limited (7).

In order to investigate the relationship between sleep disturbances and abdominal symptoms, including reflux symptoms, upper abdominal pain, fullness, constipation and diarrhea, we evaluated the results of self-reported questionnaires completed by subjects who underwent an annual health

\footnotetext{
${ }^{1}$ Second Department of Internal Medicine, Shimane University Faculty of Medicine, Japan, ${ }^{2}$ Department of Internal Medicine, Matsue Red Cross Hospital, Japan, ${ }^{3}$ Department of Preventive Medicine, Matsue Red Cross Hospital, Japan and ${ }^{4}$ Health Center, Shimane Environment and Health Public Corporation, Japan

Received for publication January 30, 2014; Accepted for publication April 24, 2014

Correspondence to Dr. Masahito Aimi, aimi@med.shimane-u.ac.jp
} 
Izumo scale for abdominal symptom-related QOL

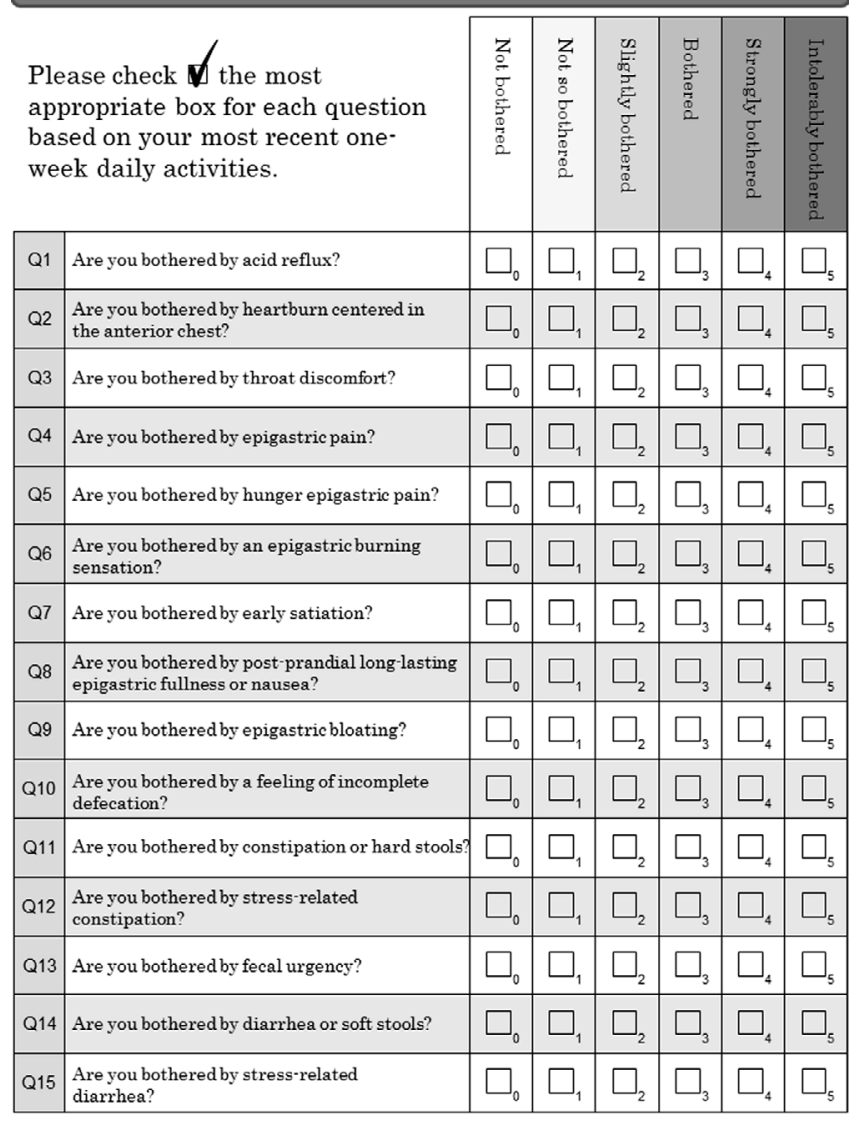

Figure 1. Izumo scale, designed to determine abdominal symptom-related quality of life impairment.

check examination.

\section{Materials and Methods}

This study was approved by the ethics committee of Matsue Red Cross Hospital. All subjects who visited the Department of Preventive Medicine of Matsue Red Cross Hospital for an annual health check examination between August 2011 and May 2012 were enrolled. After obtaining written informed consent for enrollment into the study, a precise medical history was taken. Individuals being treated with drugs that influence abdominal symptoms, such as PPIs, were excluded from this study, as were those with a history of major abdominal surgery. All subjects were examined using hematological and biochemical blood tests, abdominal ultrasonography, upper gastrointestinal barium meal studies or endoscopy and fecal occult blood tests. Individuals who showed any abnormal findings on these studies that may induce abdominal symptoms were not included in the study.

\section{Izumo scale}

The Izumo scale, a self-reported questionnaire of abdominal symptoms developed and validated by our group (8-10), was employed in this study (Fig. 1). This scale contains items for 15 symptoms in five domains: Reflux, Upper ab-
How likely are you to doze off or fall asleep in the situations described in the box below, in contrast to feeling just tired?

This refers to your usual way of life in recent times.

Even if you haven't done some of these things recently, try to work out how they would have affected you.

Use the following scale to choose the most appropriate number for each situation:



\begin{tabular}{|l|l|}
\hline Situation & $\begin{array}{c}\text { Chance of } \\
\text { dozing }\end{array}$ \\
\hline Sitting and reading & \\
\hline Watching TV & \\
\hline $\begin{array}{l}\text { Sitting, inactive in a public place } \\
\text { (e.g. a theatre or a meeting) }\end{array}$ & \\
\hline As a passenger in a car for 1 hr without a break & \\
\hline $\begin{array}{l}\text { Lying down to rest in the afternoon when } \\
\text { circumstances permit }\end{array}$ & \\
\hline Sitting and talking to someone & \\
\hline Sitting quietly after a lunch without alcohol & \\
\hline In a car, while stopped for a few minutes in the traffic & \\
\hline
\end{tabular}

Figure 2. Epworth Sleepiness Scale.

dominal pain, Fullness, Constipation and Diarrhea. The answers are rated on a six-point Likert scale from 0 to 5 , with higher values indicating a more severe impact on daily functioning. Each question is grouped into one of the five domains, with questions 1,2 and 3 grouped into the Reflux domain, questions 4,5 and 6 grouped into the Upper abdominal pain domain, questions 7,8 and 9 grouped into the Fullness domain, questions 10,11 and 12 grouped into the Constipation domain and questions 13, 14, and 15 grouped into the Diarrhea domain. This questionnaire has been shown to have good internal consistency and reproducibility, as well as correlation with a visual analogue scale of abdominal symptoms, the Gastrointestinal Symptom Rating Scale (GSRS) $(8,9)$.

When a subject recorded a score ranging from 3-5 on the Likert scale in response to a question, they were considered to have the related symptom (10). When at least one of the symptoms in a specific domain was regarded as positive, the domain was graded as positive. In this study, both positive and negative responses in each domain were evaluated in all of the enrolled subjects.

\section{Epworth Sleepiness Scale}

The Epworth Sleepiness Scale (ESS) was developed to measure subjective sleepiness (11) and contains eight questions concerning sleepiness during common daily activities, such as watching television, on a self-reported questionnaire (Fig. 2). The Japanese version of this scale was developed 


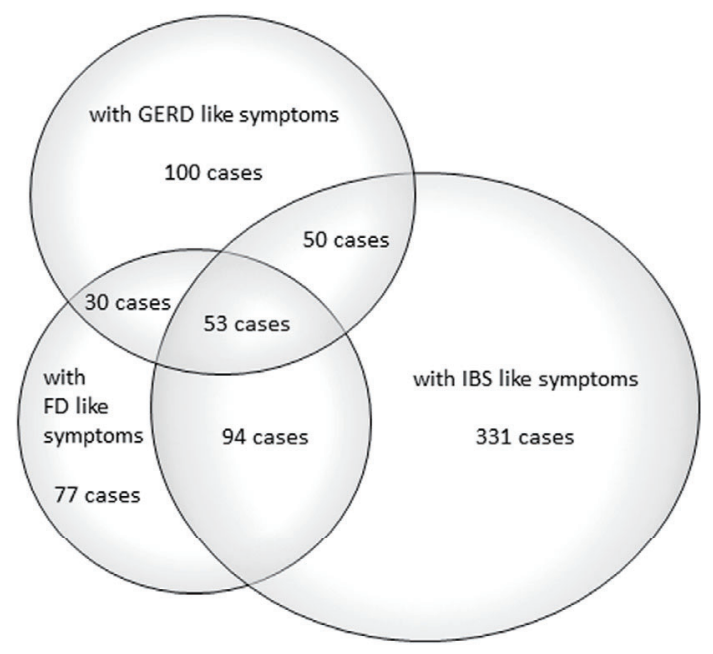

Figure 3. Overlap of GERD-, FD-, and IBS-like symptoms.

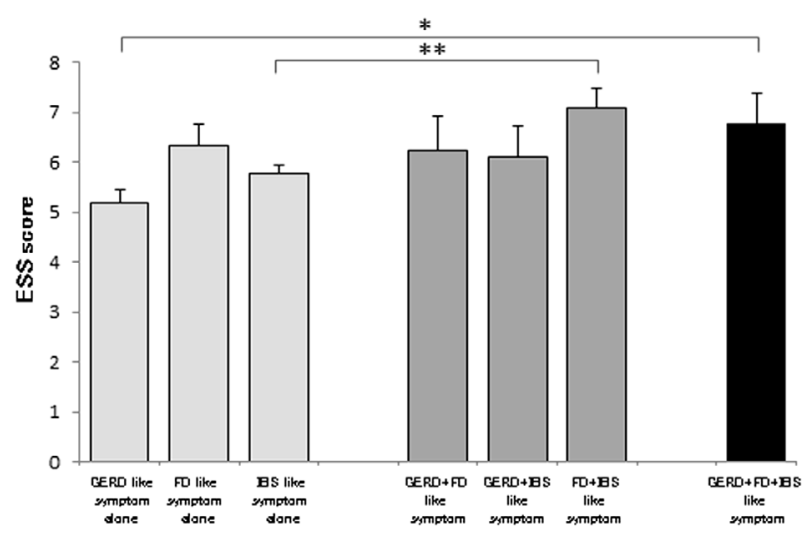

Figure 5. ESS scores in subjects with single abdominal symptom and those with multiple abdominal symptoms. Data was expressed by mean \pm S.E. $* \mathbf{p}<0.05$, significantly different. $* * \mathbf{p}<0.01$, significantly different.

and validated in 2009 (12). Answers are scored using a fourpoint Likert scale from 0 to 3, with higher values indicating a more severe impact on daily functioning. The total value of all scores is taken as the ESS score. The mean ESS score for a group of normal individuals has previously been reported to be $5.9 \pm 2.2$, while the cutoff point for sleep disturbances has been found to be between 10 and $11(11,12)$.

\section{Statistics}

The data are presented as the mean \pm S.E. The statistical analyses were performed using the chi-square test, KruskalWallis test and Mann-Whitney U-test. A multiple logistic regression analysis was also used. All calculations were performed using the Stat View 5.0 software package for Macintosh (Abacus Concepts, Berkeley, USA). Differences at a $\mathrm{p}$ value of $<0.05$ were considered to be statistically significant.

\section{Results}

We enrolled 2,936 subjects in this study, of whom 1,835

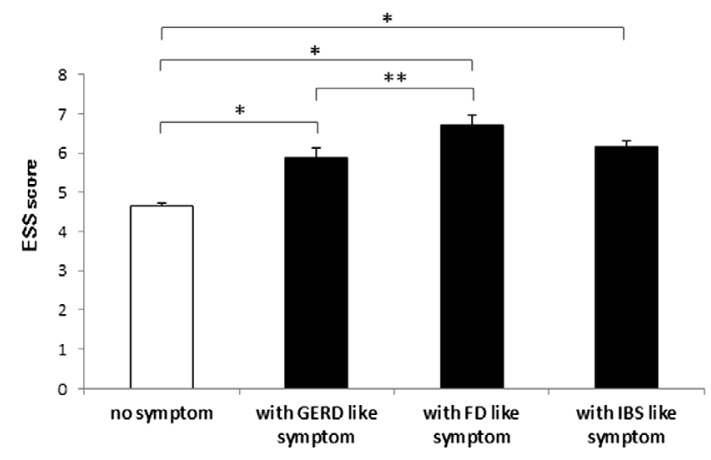

Figure 4. ESS scores in subjects with and without various abdominal symptoms. Data was expressed by mean \pm S.E. $* \mathbf{p}<0.001$, significantly different. $* * \mathbf{p}<0.05$, significantly different.

were men. The mean age of the enrolled patients was $54.8 \pm$ 0.2 years. Seventy-five percent of the subjects had no abdominal symptoms, while $25 \%$ had at least one symptom. Furthermore, 233 (7.9\% of the enrolled subjects) patients with reflux domain symptoms were classified as having GERD-like symptoms, 254 (8.6\%) with upper abdominal pain and/or fullness domain symptoms were classified as having functional dyspepsia (FD)-like symptoms and 528 (18\%) with constipation and/or diarrhea domain symptoms were classified as having irritable bowel syndrome (IBS)like symptoms. There was some overlap between the subjects with GERD-, FD- and IBS-symptoms (Fig. 3).

The ESS scores of the subjects with GERD-, FD- and IBS-like symptoms were significantly higher than those of the asymptomatic subjects. In addition, the ESS scores of the subjects with FD-like symptoms were significantly higher than those of the subjects with GERD-like symptoms (Fig. 4). Furthermore, the subjects with multiple abdominal symptoms tended to have higher ESS scores than those with single symptoms (Fig. 5).

The subjects were divided into sleep disturbed $(\geq 11$ points) and undisturbed ( $\leq 10$ points) groups based on the ESS score cutoff value $(11,12)$. A higher percentage of subjects in the sleep disturbed group complained of GERD-, FD- or IBS-like symptoms, and the number of symptomatic subjects in the sleep disturbed group was more than double that noted in the undisturbed group (Fig. 6).

When the effects of various factors on sleep disturbances (ESS score 211 ) were evaluated according to the multiple logistic regression analysis, a younger age and the presence of FD- and IBS-like symptoms were found to be significant influencing factors. Further separation of FD- and IBS-like symptoms into upper abdominal pain, fullness, constipation and diarrhea clarified that upper abdominal pain and diarrhea had a significant effect on the incidence of sleep disturbances (Table).

\section{Discussion}

Good sleep is an important factor for the quality of life 


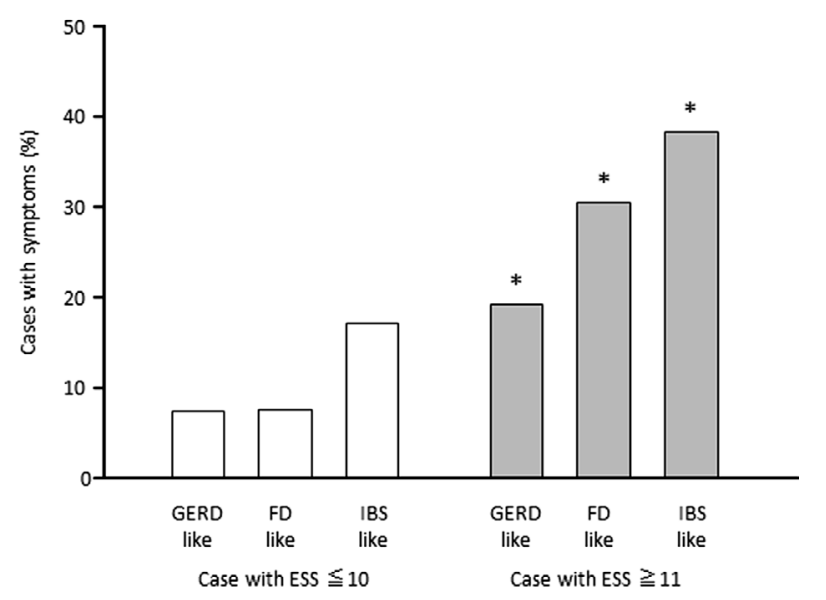

Figure 6. Percentage of subjects with each symptom. Among those with a high ESS score ( $\geq 11$ ), the percentage of symptomatic subjects was higher than that of asymptomatic subjects. *p<0.01, significantly different for each symptom.

and it may be disturbed by gastrointestinal symptoms. Reflux symptoms, including heartburn and acid regurgitation, that occur during the nocturnal period have been repeatedly reported to impair sleep quality (3-6), and GERD is regarded to be a disease with nocturnal symptoms that must be completely eliminated in order to relieve sleep disturbances (13).

In the present study, the relationships between sleep disturbances and various abdominal symptoms were evaluated in subjects without organic gastrointestinal disease. Our results showed that GERD-like symptoms, such as heartburn and acid regurgitation, aggravate daytime sleepiness, as previously reported $(3-5,13)$. However, the presence of GERDlike symptoms was not found to have a statistically significant effect on the incidence of sleep disturbances (ESS score $\geq 11$ ) according to a multiple regression analysis. On the other hand, the presence of FD- and IBS-like symptoms was identified to be a statistically significant risk factor for sleep disturbances (ESS score $\geq 11$ ) in the multiple regression analysis. In addition, upper abdominal pain and diarrhea were found to be especially related to sleep disturbances. Therefore, the presence of FD- and IBS-like symptoms is considered to have a stronger relationship with sleep disturbances than reflux symptoms.

Abdominal symptoms may decrease both sleep quantity and quality via various mechanisms, including frequent nocturnal awakening (14-17). On the other hand, sleep deprivation has been reported to enhance gastrointestinal sensitivity and aggravate unpleasant abdominal symptoms (18-20). Therefore, there may exist a causal relationship between abdominal symptoms and sleep disturbances, although the mechanisms underlying this relationship are difficult to identify.

The subjects of this study included a non-clinical population without organic gastrointestinal diseases. Therefore, the abdominal symptoms observed among our study subjects are thought to be caused by functional disorders and may not
Table. Effects of Various Factors on Daytime Sleepiness (ESS $\geq 11$ ) by Multiple Logistic Regression Analysis

\begin{tabular}{lccc}
\hline & Odds ratio & $95 \% \mathrm{CI}$ & $\mathrm{p}$ value \\
\hline Age & 0.974 & $0.959-0.989$ & $<0.001$ \\
Gender (male) & 1.304 & $0.722-1.482$ & 0.854 \\
GERD-like symptoms & 1.585 & $0.951-2.653$ & 0.074 \\
FD-like symptoms & 3.375 & $2.149-5.300$ & $<0.001$ \\
IBS-like symptoms & 1.906 & $1.272-2.856$ & $<0.001$ \\
Upper abdominal pain & 2.603 & $1.527-4.440$ & $<0.001$ \\
Fullness & 1.541 & $0.846-2.806$ & 0.157 \\
Constipation & 1.585 & $0.987-2.545$ & 0.057 \\
Diarrhea & 1.811 & $1.145-2.865$ & 0.011 \\
\hline
\end{tabular}

have been strong enough to encourage the individuals to visit a clinic for treatment. Nevertheless, our results showed that the patients with abdominal symptoms experienced significant sleep disturbances. Therefore, the abdominal symptoms of patients treated at clinics, as well as those who do not seek treatment, are expected to worsen the quantity and quality of sleep, even though such symptoms are not caused by organic gastrointestinal diseases.

There are some limitations associated with this study. For example, the presence of a sleep disturbance was evaluated according to the ESS score only. Sleep disturbances can be assessed using different tools, such as the Pittsburg Sleep Quality Index (PSQI) (21) or St. Mary's Hospital Sleep Questionnaire (SMH) (22). The PSQI is a composite questionnaire that evaluates the degree of sleep disturbance with a tendency to be influenced by the effects of an individual's mental state, particularly a depressive state (21), while the SMH is designed to detect sleep disturbances in inpatients and can also be affected by a depressive mental state (22). In contrast, the ESS was designed to primarily detect sleepiness in outpatients, and the questionnaire is easy to answer and can be used to detect the effects of sleep disturbances on daily life $(11,12)$. Therefore, we used the ESS score to evaluate the presence of sleep disturbances in our non-clinic subjects. Meanwhile, the presence of abdominal symptoms was assessed using only the Izumo scale. Although this selfreported questionnaire scale has been validated and is widely used with good reproducibility and accuracy, each questionnaire has specific characteristics $(8,9)$. Therefore, additional studies employing various parameters to evaluate sleep disturbances and abdominal symptoms are needed to confirm and expand our findings. Furthermore, in this study, we did not investigate several factors, such as the mental state, work schedule, environmental conditions or drinking and smoking habits of our subjects, which have been demonstrated to affect sleep (23-29). Therefore, further research is needed to confirm the effects of abdominal symptoms on sleep disturbances by analyzing several additional factors that affect sleep.

In conclusion, we found that, in addition to GERD symptom, FD and IBS symptoms have a strong relationship with the incidence of sleep disturbances from the viewpoint of daytime sleepiness. 
The authors state that they have no Conflict of Interest (COI).

\section{Acknowledgement}

This work was supported by a research fund from the Shimane University Faculty of Medicine.

\section{References}

1. Vakil N, van Zanten SV, Kahrilas P, Dent J, Jones R. The Montreal definition and classification of gastroesophageal reflux disease global evidence-based consensus. Am J Gastroenterology 101: 1900-1920, 2006.

2. Kusano M, Kouzu T, Kawano T, Ohara S. Nationwide epidemiological study on gastroesophageal reflux disease and sleep disorders in the Japanese population. J Gastroenterol 43: 833-841, 2008.

3. Dimarino AJ Jr, Banwait KS, Eschinger E, et al. The effect of gastro-oesophageal reflux and omeprazole on key sleep parameters. Aliment Pharmacol Ther 22: 325-329, 2005.

4. Johnson DA, Orr WC, Crawley JA, et al. Effect of esomeprazole on nighttime heartburn and sleep quality in patients with GERD: a randomized, placebo-controlled trial. Am J Gastroenterol 100: 1914-1922, 2005

5. Fujiwara Y, Kohata Y, Kaji M, et al. Sleep dysfunction in Japanese patients with gastroesophageal reflux disease: prevalence, risk factors, and efficacy of rabeprazole. Digestion 81: 135-141, 2010.

6. Fujiwara Y, Arakawa T, Fass R. Gastroesophageal reflux disease and sleep disturbances. J Gastroenterol 47: 760-769, 2012.

7. Shaker R, Castell DO, Schoenfeld PS, Spechler SJ. Nighttime heartburn is an under-appreciated clinical problem that impacts sleep and daytime function: the results of a Gallup survey conducted on behalf of the American Gastroenterological Association. Am J Gastroenterol 98: 1487-1493, 2003.

8. Furuta $\mathrm{K}$, Ishihara $\mathrm{S}$, Sato $\mathrm{S}$, et al. Development and verification of the Izumo Scale, new questionnaire for quality of life assessment of patients with gastrointestinal symptoms. Nihon Shokakibyo Gakkai Zasshi (Japanese Journal of Gastroenterology) 106: 1478-1487, 2009 (in Japanese, Abstract in English).

9. Furuta $\mathrm{K}$, Ishihara $\mathrm{S}$, Sato $\mathrm{S}$, et al. Verification of responsiveness in the Izumo Scale for patients with gastrointestinal symptoms. Ther Res 30: 1651-1658, 2009 (in Japanese).

10. Kakuta E, Yamashita N, Katsube T, et al. Abdominal symptomrelated QOL in individuals visiting an outpatient clinic and those attending an annual health check. Intern Med 50: 1517-1522, 2011

11. Johns MW. A new method for measuring daytime sleepiness: the Epworth sleepiness scale. Sleep 14: 540-545, 1991.

12. Takegami M, Suzukamo Y, Wakita T, et al. Development of a
Japanese version of the Epworth Sleepiness Scale (JESS) based on item response theory. Sleep Med 10: 556-565, 2009.

13. Orr WC. Review article: sleep-related gastro-oesophageal reflux as a distinct clinical entity. Aliment Pharmacol Ther 31: 47-56, 2010.

14. Dickman R, Green C, Fass SS, et al. Relationships between sleep quality and $\mathrm{pH}$ monitoring findings in persons with gastroesophageal reflux disease. J Clin Sleep Med 3: 505-513, 2007.

15. Orr WC, Robinson MG, Johnson LF. The effect of esophageal acid volume on arousals from sleep and acid clearance. Chest 99: 351-354, 1991.

16. Orr WC, Johnson LF. Responses to different levels of esophageal acidification during waking and sleep. Dig Dis Sci 43: 241-245, 1998.

17. Heitkemper M, Jarrett M, Burr R, et al. Subjective and objective sleep indices in women with irritable bowel syndrome. Neurogastroenterol Motil 17: 523-530, 2007.

18. Goldsmith G, Jeffrey SL. Effect of sleep quality on symptoms of irritable bowel syndrome. Dig Dis Sci 38: 1809-1814, 1993.

19. Tally NJ, Phillips SF, Melton LJ, Mulvihill C, Wiltgen C, Zinsmeister AR. Diagnostic value of the Manning criteria in irritable bowel syndrome. Gut 31: 77-81, 1990.

20. Jarrett M, Heitkemper M, Carin KC, Burr RL, Hertig V. Sleep disturbance influences gastrointestinal symptoms in women with irritable bowel syndrome. Dig Dis Sci 45: 952-959, 2000.

21. Buysse DJ, Reynolds CF 3rd, Monk TH, Berman SR, Kupfer DJ. The Pittsburgh Sleep Quality Index: a new instrument for psychiatric practice and research. Psychiatry Res 28: 193-213, 1989.

22. Ellis BW, Jones MW, Lancaster R, Raptopoulos P, Angelopoulos N, Priest RG. The St Mary's Hospital sleep questionnaire: a study of reliability. Sleep 4: 93-97, 1981.

23. Glozier N, Davenport T, Hickie IB. Identification and management of depression in Australian primary care and access to specialist mental health care. Psychiatr Serv 14: 1247-1251, 2012.

24. Crowley SJ, Acebo C, Carskadon MA. Sleep, circadian rhythms, and delayed phase in adolescence. Sleep Med 14: 602-612, 2007.

25. Kerkhof GA. The 24-hour variation of mood differs between morning- and evening-type individuals. Percept Motor Skills 14: 264-266, 1998.

26. Wetter DW, Young TB. The relation between cigarette smoking and sleep disturbance. Prevent Med 14: 328-334, 1994.

27. Jaehne A, Loessl B, Bárkai Z, Riemann D, Hornyak M. Effects of nicotine on sleep during consumption, withdrawal and replacement therapy. Sleep Med Rev 14: 363-377, 2009.

28. Ebrahim IO, Shapiro CM, Williams AJ, Fenwick PB. Alcohol and sleep I: effects on normal sleep. Alcohol Clin Exp Res 14: 539549, 2013.

29. Van Reen E, Rupp TL, Acebo C, Seifer R, Carskadon MA. Biphasic effects of alcohol as a function of circadian phase. Sleep 14: 137-145, 2013.

(C) 2014 The Japanese Society of Internal Medicine http://www.naika.or.jp/imonline/index.html 